\title{
Decoupling of lattice and orbital degrees of freedom in an iron-pnictide superconductor
}

\author{
C. E. Matt $\odot,{ }^{1,2,{ }^{*}}$ O. Ivashko, ${ }^{2}$ M. Horio $\odot,{ }^{2}$ J. Choi, ${ }^{2}$ Q. Wang $\odot,{ }^{2}$ D. Sutter, ${ }^{2}$ N. Dennler $\odot,{ }^{2}$ M. H. Fischer $\odot,{ }^{2}$ S. Katrych $\odot,{ }^{3}$ \\ L. Forro, ${ }^{3}$ J. Ma, ${ }^{1, \dagger}$ B. Fu, ${ }^{1}$ B. Q. Lv, ${ }^{1}$ M. v. Zimmermann, ${ }^{4}$ T. K. Kim $\odot,{ }^{5}$ N. C. Plumb, ${ }^{1}$ N. Xu, ${ }^{1}$ M. Shi, ${ }^{1}$ and J. Chang ${ }^{2}$ \\ ${ }^{1}$ Swiss Light Source, Paul Scherrer Institut, CH-5232 Villigen PSI, Switzerland \\ ${ }^{2}$ Physik-Institut, Universität Zürich, Winterthurerstrasse 190, CH-8057 Zürich, Switzerland \\ ${ }^{3}$ Laboratory of Physics of Complex Matter, École Polytechnique Fédérale de Lausanne (EPFL), CH-1015 Lausanne, Switzerland \\ ${ }^{4}$ Deutsches Elektronen-Synchrotron DESY, 22603 Hamburg, Germany \\ ${ }^{5}$ Diamond Light Source, Harwell Campus, Didcot OX11 ODE, United Kingdom
}

(Received 11 October 2020; accepted 11 May 2021; published 21 June 2021)

\begin{abstract}
The interplay between structural and electronic phases in iron-based superconductors is a central theme in the search for the superconducting pairing mechanism. While electronic nematicity is competing with superconductivity, the effect of purely structural orthorhombic order is unexplored. Here, using x-ray diffraction and angle-resolved photoemission spectroscopy, we reveal a structural orthorhombic phase in the electrondoped iron-pnictide superconductor $\mathrm{Pr}_{4} \mathrm{Fe}_{2} \mathrm{As}_{2} \mathrm{Te}_{0.88} \mathrm{O}_{4}\left(T_{\mathrm{c}}=25 \mathrm{~K}\right)$, which is distinct from orthorhombicity in the nematic phase in underdoped pnictides. Despite the high electron doping we find an exceptionally high orthorhombic onset temperature $\left(T_{\text {ort }} \sim 250 \mathrm{~K}\right)$, no signatures of phase competition with superconductivity, and absence of electronic nematic order as the driving mechanism for orthorhombicity. Combined, our results establish a high-temperature phase in the phase diagram of iron-pnictide superconductors and impose strong constraints for the modeling of their superconducting pairing mechanism.
\end{abstract}

DOI: 10.1103/PhysRevResearch.3.023220

\section{INTRODUCTION}

Many iron pnictides display an intrinsic susceptibility towards fourfold symmetry breaking in structural, spin, and electronic degrees of freedom (DOF) [1,2]. A well-known and heavily investigated example is the nematic state, located in the underdoped range of the iron-pnictide phase diagram. Due to its close proximity to superconductivity and its putative quantum criticality [3-5], the nematic phase has been under scrutiny to uncover the microscopic description of the superconducting pairing mechanism. Among other experiments, transport [6-8] and angle-resolved photoemission spectroscopy (ARPES) [9-14] studies have provided compelling evidence that nematicity in underdoped iron pnictides is triggered by an electronic order that subsequently breaks the lattice fourfold rotational (C4) symmetry [15], as illustrated in Figs. 1(a) and 1(b). In ARPES experiments, the orbital order was directly revealed by the splitting of the $d_{x z}-d_{y z}$ bands, which are degenerate in the high-temperature tetragonal phase [see Fig. 1(b)]. In some parent compounds, a band splitting of up to $\sim 60 \mathrm{meV}$ was observed [9-12], which is

\footnotetext{
*cmatt@g.harvard.edu

Current Address: Department of Physics, City University of Hong Kong, Kowloon, Hong Kong, China.
}

Published by the American Physical Society under the terms of the Creative Commons Attribution 4.0 International license. Further distribution of this work must maintain attribution to the author $(s)$ and the published article's title, journal citation, and DOI. too large to be a trivial consequence of the small orthorhombicity of less than $1 \%$ (Ref. [16]). This led to the conclusion that electronic order is driving the nematic state in underdoped pnictides [17], triggering a tetragonal-to-orthorhombic transition in the lattice DOF at the same temperature scale $\left(T_{\text {ort }}=T_{\text {nem }}\right)$. Importantly, x-ray diffraction (XRD) measurements of the orthorhombic distortion revealed that the electronically driven nematic phase is competing with superconductivity $[15,18]$. However, due to the strong coupling of electronic and lattice DOF, the competing channel (i.e., electronic or structural) is ambiguous. This calls for a disentanglement of structurally from electronically broken symmetries and investigation of their separate effects on superconductivity. Revealing new phases which show less entangled orders in structural, spin, and electronic DOF is therefore a promising avenue to solve this pressing problem.

Here we report the observation of an orthorhombic phase in the electron-doped iron-pnictide superconductor $\mathrm{Pr}_{4} \mathrm{Fe}_{2} \mathrm{As}_{2} \mathrm{Te}_{0.88} \mathrm{O}_{4}$ (PFATO, $T_{\mathrm{c}}=25 \mathrm{~K}$ ) [19-21]. Our XRD study reveals that this phase has an exceptionally high onset temperature of $T_{\text {ort }}=250 \mathrm{~K}$. This is $\sim 50 \mathrm{~K}$ higher than the maximum $T_{\text {ort }}$ which has so far been observed in iron-based superconductors [2]. Considering the high electron doping of 0.12 electrons per $\mathrm{Fe}$, this observation is unexpected. Furthermore, we find that the low-temperature structural orthorhombic order parameter $\delta$ is not suppressed below $T_{\mathrm{c}}$, indicating a "friendly" coexistence of orthorhombicity with superconductivity, as also observed in FeSe under pressure [22]. The structural (phononic) origin of the orthorhombic phase is further supported by our ARPES measurements, which reveal the absence of orbital splitting down to the 
(a)

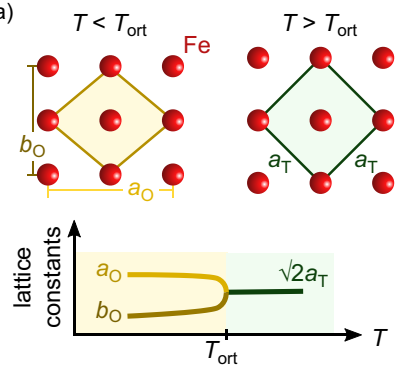

(b)

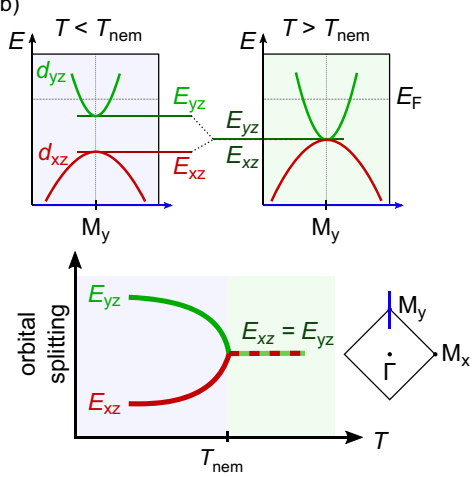

(c)

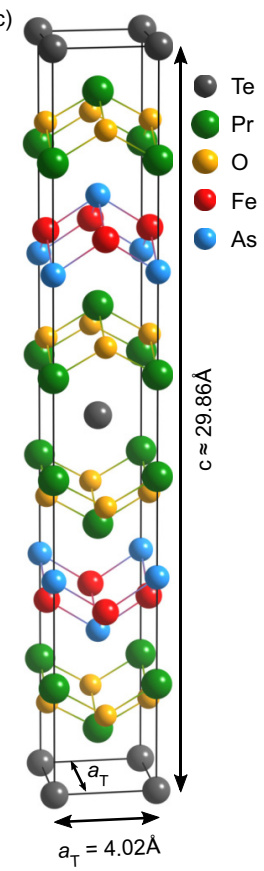

(d)

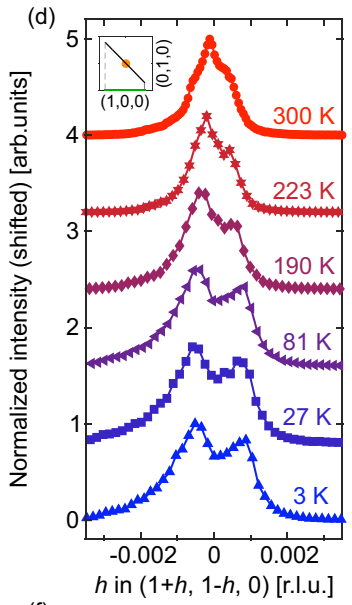

(f)

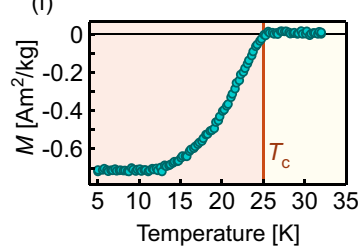

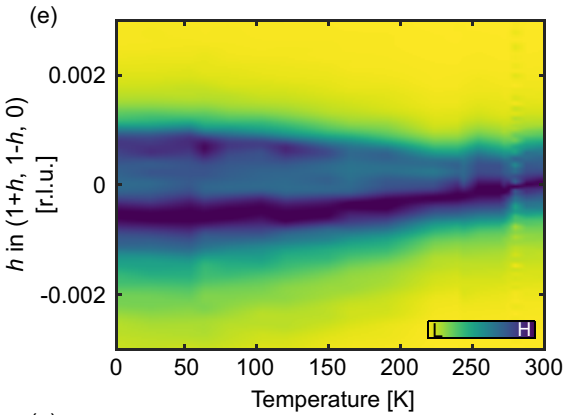

(g)

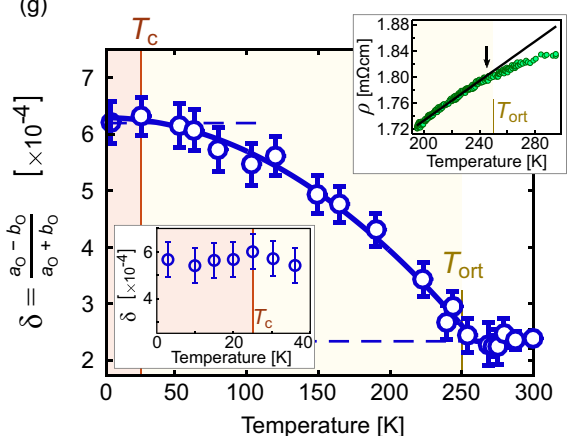

FIG. 1. High-temperature orthorhombicity in $\mathrm{Pr}_{4} \mathrm{Fe}_{2} \mathrm{As}_{2} \mathrm{Te}_{0.88} \mathrm{O}_{4}$ (PFATO). (a) Schematics for structural $\mathrm{C}_{4}$ symmetry breaking at $T<T_{\text {ort }}$ in iron pnictides. (b) Schematics of orbital splitting in the nematic phase of underdoped iron pnictides. Below $T_{\text {nem }}$, the $d_{x z}$ and $d_{y z}$ are no longer degenerate and split in energy, with largest splitting at the $\mathrm{M}$ points of the Brillouin zone [17]. Here, $E_{\mathrm{xz}}\left(E_{\mathrm{yz}}\right)$ denote the maximum (minimum) of the holelike (electronlike) bands at $\mathbf{M}_{\mathrm{y}}$. (c) Room-temperature unit cell of PFATO [19]. (d)-(e) High-resolution transverse scans through the $(1,1,0)$ Bragg reflection (see inset) at temperatures as indicated. A splitting of the diffraction peak is observed below $T_{\text {ort }} \sim 250 \mathrm{~K}$. (f) Magnetic susceptibility at $1 \mathrm{mT}$ cooled in zero field. (g) Temperature dependence of the orthorhombic order parameter $\delta$. Lower inset: $\delta$ across $T_{\mathrm{c}}$ measured in finer temperature steps. Upper inset: Resistivity vs temperature, displaying a saturation above the onset of orthorhombicity.

lowest measurable temperatures. Our ARPES results further reveal the absence of hole pockets around $\Gamma$, challenging nesting-based theories for superconductivity and spin-density wave order. These properties are orthogonal to what has been found for the nematic phase in underdoped iron pnictides and therefore indicate that this orthorhombic phase is distinct from previously described phases in iron pnictides.

\section{METHODS}

High-quality single crystals of $\mathrm{Pr}_{4} \mathrm{Fe}_{2} \mathrm{As}_{2} \mathrm{Te}_{0.88} \mathrm{O}_{4}$ were synthesized by high-pressure flux growth, as described in Ref. [19]. The tellurium off-stoichiometry provides $12 \%$ electron doping and superconductivity with a $95 \% \pm 5 \%$ volume fraction [19]. We selected the largest crystals- $50 \times$ $50 \times 10 \mu \mathrm{m}^{3}$-for ARPES and XRD experiments, respectively. Our XRD experiment was carried out on the P07 beamline at PETRA III at DESY in Hamburg. The crystal mosaicity yields additional shoulders in the room-temperature $(300 \mathrm{~K})$ rocking curve in Fig. $1(\mathrm{~d})$. Due to the small sample mass, the profile deviates from a Gaussian line shape [23]. ARPES experiments were performed at the SIS [24] and I05 beamlines [25]—Swiss Light Source, Switzerland, and Diamond, UK, respectively. The effective angular and energy resolution (standard Gaussian deviation) were set to $\sim 0.1^{\circ}$ and $\sigma_{R}=5 \mathrm{meV}$. Pristine surfaces were obtained through a top post cleaving method. Angle-dependent x-ray photoelectron spectroscopy measurements suggest that the cleaving plane is the charge-neutral Te layer (see Supplemental Material, Fig. S6). Thermal and electrical grounding were realized through a combination of silver epoxy and graphite paste. We estimated the Fermi level from reference spectra of gold or copper in electrical and thermal contact with the PFATO sample. Using density functional theory (DFT) as implemented in the WIEN2K package [26,27], we calculated the band structure of stoichiometric $\mathrm{Pr}_{4} \mathrm{Fe}_{2} \mathrm{As}_{2} \mathrm{TeO}_{4}$ (details are provided in [23]).

\section{RESULTS}

\section{A. X-ray diffraction}

We reveal the tetragonal-to-orthorhombic phase transition in PFATO by XRD measurements of the $(1,1,0)$ Bragg reflection. At room temperature, XRD studies have previously shown that PFATO has a tetragonal $14 / \mathrm{mmm}$ structure with a large $c$ axis (29.86 $\AA$ ) lattice parameter [19] [see Fig. 1(c)]. As shown in Figs. $1(\mathrm{~d})-1(\mathrm{e})$, below $T_{\text {ort }}=250 \mathrm{~K}$, the $(1,1,0)$ reflection splits into two peaks along the transverse direction, evidencing the existence of orthorhombicity. However, the onset temperature is $\sim 50 \mathrm{~K}$ higher than the highest structural transition temperature in any other iron-pnictide parent compound [2]. In light of the rather high electron doping of $\mathrm{Pr}_{4} \mathrm{Fe}_{2} \mathrm{As}_{2} \mathrm{Te}_{0.88} \mathrm{O}_{4}$ (0.12 electrons per Fe [23]), such a high onset temperature is surprising.

By analyzing the structural peak splitting [28], we infer the orthorhombic order parameter $\delta=\left(a_{\mathrm{O}}-b_{\mathrm{O}}\right) /\left(a_{\mathrm{O}}+b_{\mathrm{O}}\right)$, 

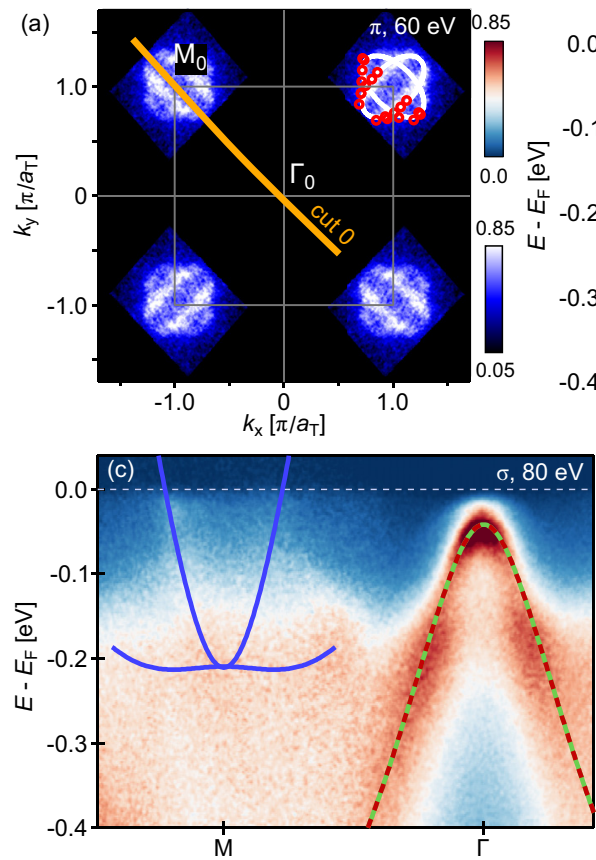
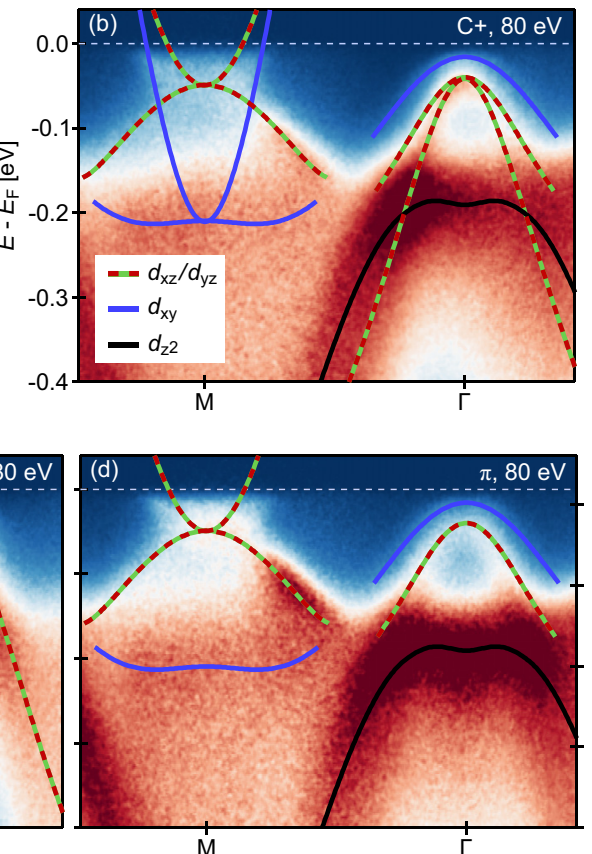

(e)

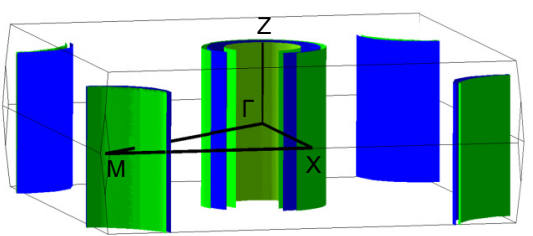

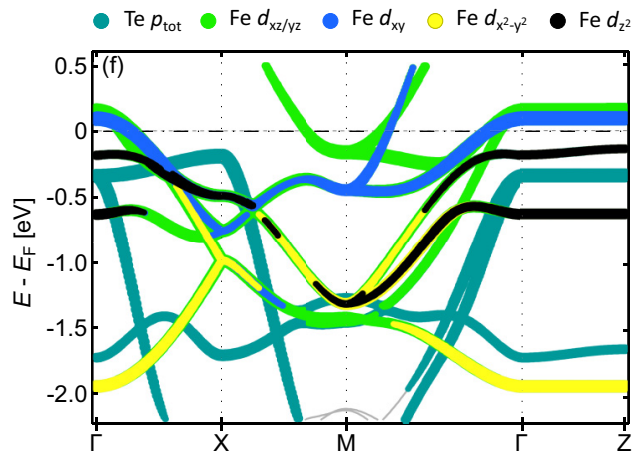

FIG. 2. Electronic band structure of $\mathrm{Pr}_{4} \mathrm{Fe}_{2} \mathrm{As}_{2} \mathrm{Te}_{0.88} \mathrm{O}_{4}$. (a) Symmetrized Fermi surface map recorded on PFATO around zone corners and displayed in false color scale with color saturation as indicated (maximum intensity is normalized to 1). The Fermi surface contours of the two electron pockets are shown schematically, with solid lines in the top right zone corner. The two underlying Fermi pockets amount to a combined filling of $\sim 0.11 \pm 0.01$ electrons per Fe [23]. (b)-(d) Low-energy electronic band structure along cut $0\left(\Gamma_{0}-\mathrm{M}_{0}\right)$ measured with circular, $\sigma$, and $\pi$ polarized light $(h v=80 \mathrm{eV})$. Overlaid lines are shifted and renormalized DFT calculated bands. All bands are visible with circular polarized light, while linear polarization probes bands depending on their orbital character, (see Supplemental Material, Fig. S1). All spectra were recorded at $5.5 \mathrm{~K}$. (e) DFT-calculated Fermi surface and (f) band structure of undoped $\mathrm{Pr}_{4} \mathrm{Fe}_{2} \mathrm{As}_{2} \mathrm{TeO}_{4}$ in which the two electron pockets around $\mathrm{M}$ compensate the three hole pockets around $\Gamma$.

where $a_{\mathrm{O}}$ and $b_{\mathrm{O}}$ are the orthorhombic lattice parameters [see Fig. 1(a)]. As shown in Fig. 1(g), $\delta$ gradually increases and plateaus below $\sim 50 \mathrm{~K}$ at $6 \times 10^{-4}$. Importantly, $\delta$ retains its maximum value for all temperatures below $T_{\mathrm{c}}$ (within error bars) indicating "friendly" coexistence with superconductivity [see lower left inset of Fig. 1(g)]. This is in contrast to the strong phase competition between the electronically induced nematic phase of underdoped iron pnictides in which nematicity gets suppressed below $T_{\mathrm{c}}$ and $\mathrm{C}_{4}$ rotational symmetry restores at low temperatures close to optimal doping [15]. It has been suggested that the suppression of orthorhombicity in the electronic nematic phase is indirect and arises due to the competition for the same electronic states between magnetism and superconductivity [1,29]. This suggests that orthorhombicity in PFATO is not triggered by electronic DOF, which compete with superconductivity.

\section{B. Angle-resolved photoemission spectroscopy}

To reveal the absence of rotational symmetry breaking in the electronic DOF, we performed high-resolution ARPES measurements on twinned crystals. It is worth noting that due to the very small sample size of $\leqslant$ $50 \times 50 \mu \mathrm{m}^{2}$, the usual methods, such as in-plane resistivity anisotropy on detwinned samples, are not feasible. However, as ARPES directly accesses the electronic structure in momentum space, it presents a suitable tech- nique to measure the electronic nematic order parameter on twinned samples by resolving the $d_{x z}-d_{y z}$ orbital splitting [9].

Figures 2(a)-2(d) show the Fermi surface and representative diagonal cuts through the tetragonal Brillouin zone (BZ) center $(\Gamma)$ and corner $(\mathrm{M})$. Vertical $(\sigma)$ and horizontal $(\pi)$ linear light polarizations are used to enhance bands of different orbital character (see Supplemental Material, Fig. S1). Furthermore, we use density functional theory calculations to assign the orbital character of each measured band, as illustrated in Figs. 2(e) and 2(f), and Supplemental Material Fig. S1. Colors of the overlaid lines indicate the main orbital character of the DFT-derived bands. Figure 3(a) summarizes the extracted electronic band structure along the $\Gamma-\mathrm{M}$ highsymmetry cut. Due to the very large $c$-axis lattice constant we expect a weak out-of-plane dispersion, which is confirmed by our DFT calculations.

Electronic nematicity manifests as an energy splitting of the $d_{x z}$ and $d_{y z}$ orbitals, as sketched in Figs. 1(b) and 3 (b). We disentangle the bands of different orbital character by using light polarization analysis on a twinned sample [Figs. 3(c)-3(n), and Supplemental Material Figs. S1-S6]. Using $\pi$ - and $\sigma$-polarized light, we observe two electronlike bands at around $\mathrm{M}_{1}$, which we identify as the $d_{x y}$ band (blue) and a superposition of the $d_{x z}$ and $d_{y z}$ bands [see Figs. 3(c)3(f)]. A cut through $\mathrm{M}_{0}$ with circular polarized light is shown in Figs. 3(g) and 3(h). We observe the $d_{x z}-d_{y z}$ holelike 

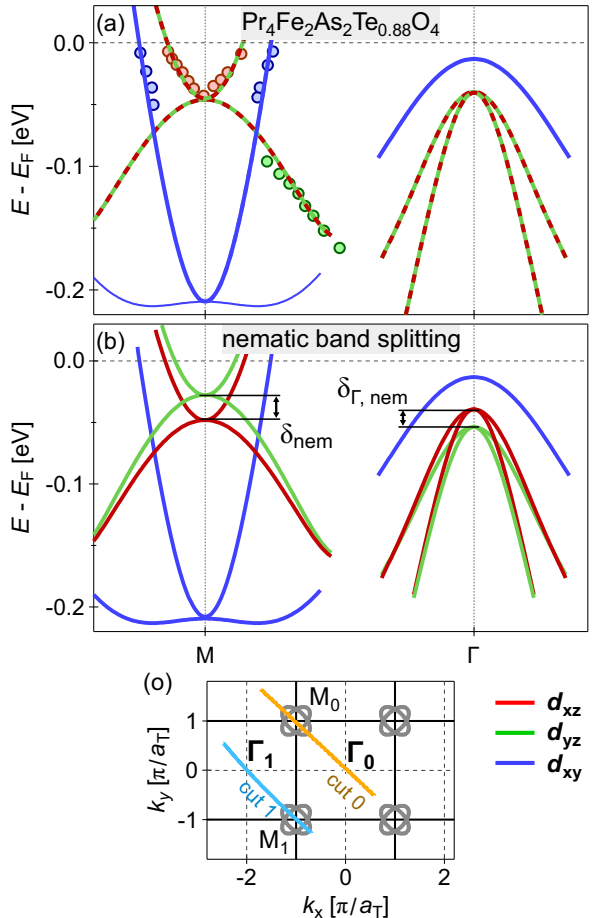
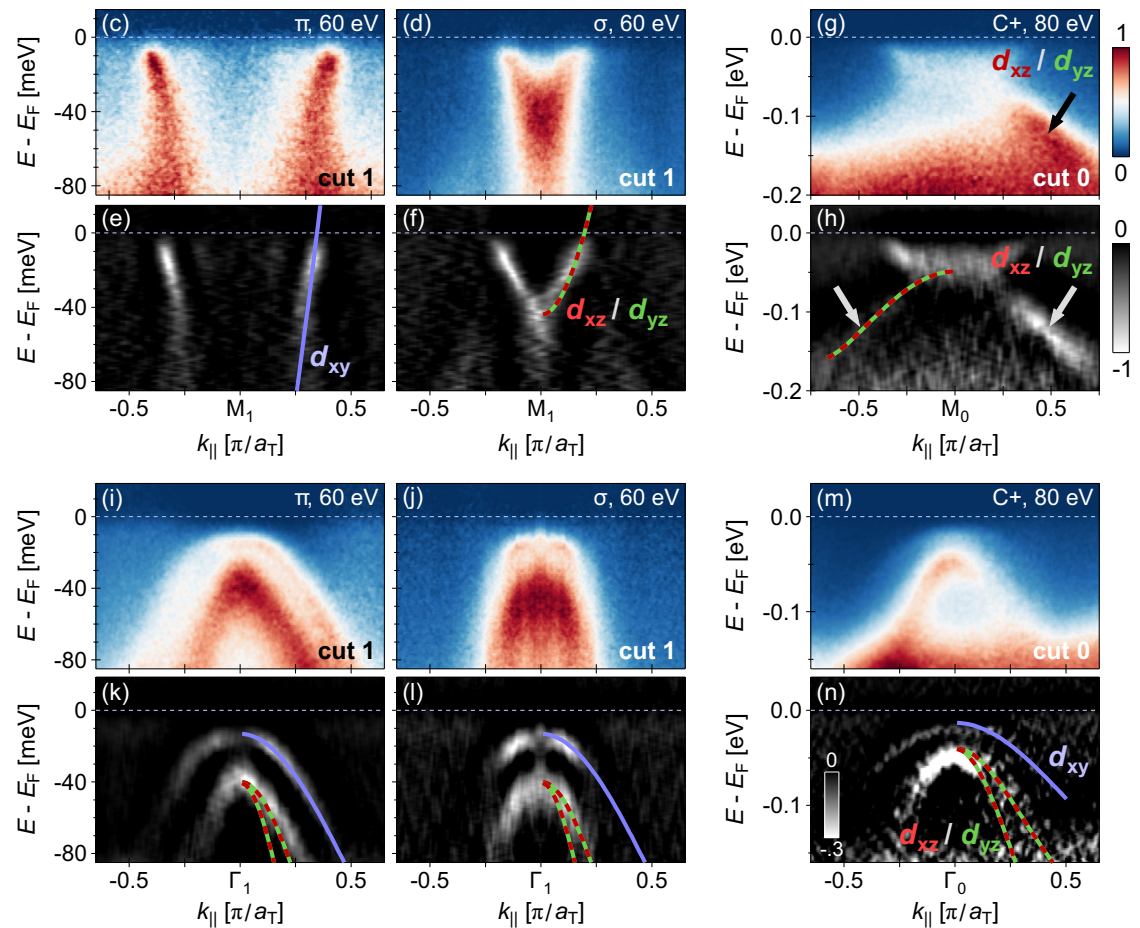

FIG. 3. Absence of electronic nematicity in $\mathrm{Pr}_{4} \mathrm{Fe}_{2} \mathrm{As}_{2} \mathrm{Te}_{0.88} \mathrm{O}_{4}$ (PFATO). (a) Schematics of observed band dispersion in PFATO. (b) Schematics of band dispersion expected for the electronic nematic phase with a finite nematic order parameter (energy splitting between $d_{x z}$ and $d_{y z}$ states) of $\delta_{\text {nem }} \sim 20 \mathrm{meV}$. (c)-(h) Diagonal cuts through $\mathrm{M}_{0}$ and $\mathrm{M}_{1}$, defined in panel (o), measured at $5.5 \mathrm{~K}$ with photon energy and polarization as indicated, plotted in false-color scale with color saturation as indicated (intensity normalized to 1). (e), (f), (h) Curvature plots of the raw spectra (c), (d), and (g) calculated as described in Ref. [30] (minimum intensity renormalized to -1). All expected bands with $d_{x y}, d_{x z}$, and $d_{y z}$ orbital character (blue, red, and green lines) are identified. (i)-(n) Similar spectra as (c)-(h) but for cuts through $\Gamma_{0}$ and $\Gamma_{1}$, as indicated in (o). Spectra in (j) and (l) are symmetrized around $\Gamma_{1}$. (o) Sketch of the Brillouin zone and electronlike Fermi surface sheets indicated in gray colors and cut 0 and cut 1 indicated in orange and blue. Horizontal dashed lines in (a)-(n) indicate the Fermi level $E_{\mathrm{F}}$.

band at binding energy of $E_{\mathrm{B}} \sim-100 \mathrm{meV}$. Note that the $d_{x y}$ band at $\mathrm{M}$ is clearly separated from the $d_{x z}-d_{y z}$ bands, as it disperses down to much lower energies. This allows us to unambiguously disentangle them and avoids complications as found in the nematic state of FeSe, where all three bands are much closer to one another [31,32].

Figures 3(i)-3(n) depict the bands around the $\Gamma$ point in the first and second BZ, probed by different light polarization. We observe the top of the three holelike bands with each polarization. The inner two $d_{x z}-d_{y z}$ bands are degenerate at $\Gamma$ but disperse at a different velocity such that they can be clearly disentangled at nonzero momenta. Their degeneracy at $\Gamma$ agrees with our orbital assignment based on their polarization dependence (see Supplemental Material, Figs. S1 and S3). The outermost holelike band $\left(d_{x y}\right)$ is distinct from the inner two bands at all momenta around $\Gamma$. For all three light polarizations and around both the $\mathrm{M}$ and $\Gamma$ point, we observe that the $d_{x z}$ and $d_{y z}$ bands are degenerate in the raw ARPES spectra as well as in the corresponding curvature plots. This indicates negligible nematic band splitting [32,33] at lowest measured temperatures and in the normal state (see Supplemental Material, Figs. S4 and S5).

\section{DISCUSSION}

In doped iron pnictides, orthorhombicity, that is triggered by electronic nematicity, is strongly suppressed below $T_{\mathrm{c}}$ for similarly sized $\delta \sim 6 \times 10^{-4}$ (see inset of Fig. $4[15,18]$ ). Such behavior signals a pronounced phase competition, as illustrated by blue dashed line in Fig. 4. In PFATO $\delta$ is constant across $T_{\mathrm{c}}$, demonstrating "friendly" phase coexistence, and suggesting that orthorhombicity is not triggered by electronic nematicity.

To further demonstrate the absence (negligibility) of electronic nematicity, we compare the dominating energy scales in PFATO. PFATO shows remarkably strong superconductivity with a superconducting gap size of $\Delta_{\mathrm{sc}} \sim 12 \mathrm{meV}$ (see Supplemental Material, Fig. S7). If nematicity was triggering orthorhombicity, we would expect its energy scale to be dominating over $\Delta_{\mathrm{sc}}$ due to (i) the absence of suppression of $\delta$, and (ii) the record-high orthorhombic transition temperature. In such a scenario we would expect the nematic band splitting to be considerably larger than $\Delta_{\mathrm{sc}} \sim 12 \mathrm{meV}$. Our applied energy resolution sets a lower bound of $12 \mathrm{meV}$ for the possible nematic band splitting. Any splitting larger than $12 \mathrm{meV}$ would be resolved (see Supplemental Material, Fig. $\mathrm{S} 8$ ). We therefore conclude that the orthorhombic phase is not triggered by electronic nematicity and is of different origin.

\section{CONCLUSION}

In combination our XRD and ARPES study on the ironbased superconductor PFATO provide strong evidence for a different phase in the iron-pnictide phase diagram. The 


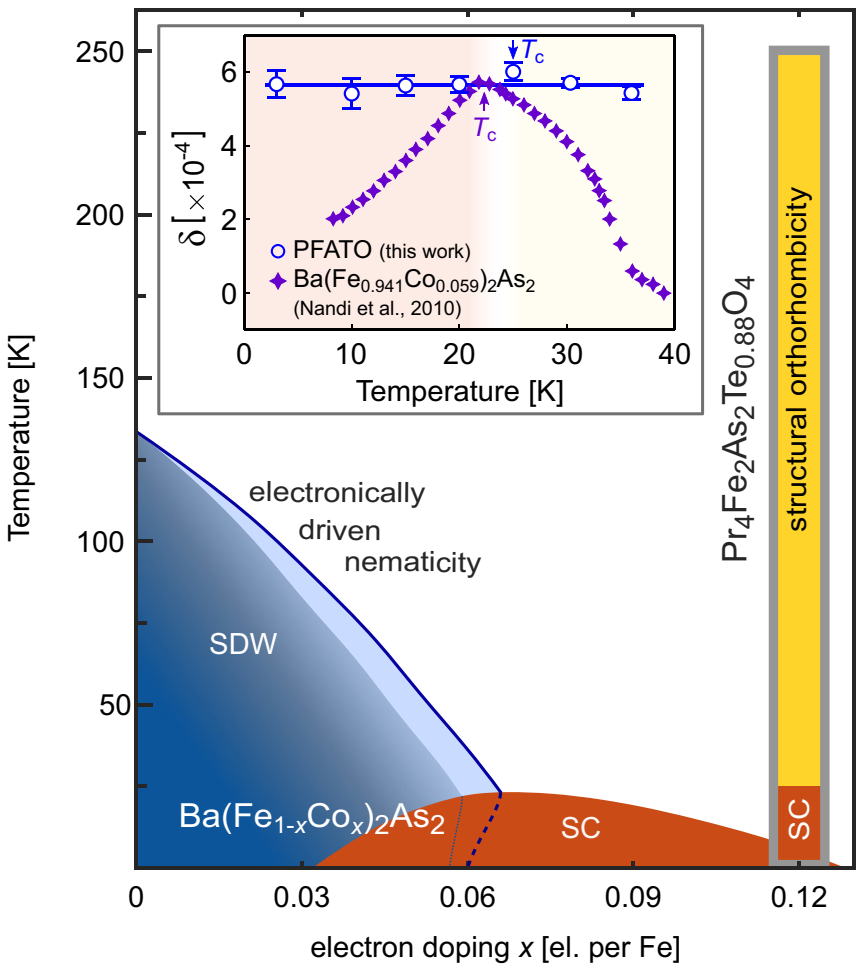

FIG. 4. Comparison between $\mathrm{Pr}_{4} \mathrm{Fe}_{2} \mathrm{As}_{2} \mathrm{Te}_{0.88} \mathrm{O}_{4}$ and $\mathrm{Ba}\left(\mathrm{Fe}_{1-x} \mathrm{Co}_{x}\right)_{2} \mathrm{As}_{2}$. The nematic and spin-density wave (SDW) phases in underdoped $\mathrm{Ba}\left(\mathrm{Fe}_{1-x} \mathrm{Co}_{x}\right)_{2} \mathrm{As}_{2}$ follow a similar doping dependence and are intersecting the superconducting dome close to optimal doping at $\sim 0.06$ electrons per Fe. At roughly twice the doping $(x=0.12), \mathrm{Pr}_{4} \mathrm{Fe}_{2} \mathrm{As}_{2} \mathrm{Te}_{0.88} \mathrm{O}_{4}$ hosts a high-temperature onset of orthorhombicity at $T_{\text {ort }} \sim 250 \mathrm{~K}\left[100 \mathrm{~K}\right.$ above $T_{\text {nem }}$ of $\mathrm{BaFe}_{2} \mathrm{As}_{2}$ ], and a superconducting transition at $T_{c} \sim 25 \mathrm{~K}$ [similar to optimally doped $\left.\mathrm{Ba}\left(\mathrm{Fe}_{1-x} \mathrm{Co}_{x}\right)_{2} \mathrm{As}_{2}\right]$. Inset: Orthorhombic order parameter versus temperature for $\mathrm{Ba}\left(\mathrm{Fe}_{0.941} \mathrm{Co}_{0.059}\right)_{2} \mathrm{As}_{2}$ and PFATO. The partial (absence of) suppression below $T_{c}$ evidences phase competition ("friendly" coexistence) between orthorhombicity/nematicity and superconductivity. Blue line is a linear fit to $\delta$.

direct comparison between PFATO and the widely studied $\mathrm{Ba}\left(\mathrm{Fe}_{1-x} \mathrm{Co}_{x}\right)_{2} \mathrm{As}_{2}$ system (Fig. 4) highlights the difference between the electronic nematic phase and the here discussed structural orthorhombic phase from the perspective of doping dependence. The phase transition of the electronic nematic phase has its highest $T_{\text {nem }}$ at the parent compound, which is then decaying once electrons are doped into the system. Furthermore, it is closely accompanied by a spin-density-wave (SDW) phase, which follows a similar doping-dependence. So far, a magnetic transition has not been found in PFATO [19-21] but could possibly be stabilized under pressure, similar to bulk FeSe [22]. Furthermore, the absence of SDW order could be explained by the poorly fulfilled nesting condition, due to the absence of the holelike Fermi surface around $\Gamma$ [see Fig. 2(a)]. From this standpoint it is inconceivable that the orthorhombic phase of $12 \%$ electron-doped PFATO has a similar doping dependence and is of the same origin as the nematic phase. PFATO therefore presents an iron pnictide with a purely structural phase transition, with electronic DOF unaffected, preceding the superconducting dome. The structural phase transition is likely driven by phonon modes, which makes this system highly interesting for studying the influence of lattice vibrations on the superconducting pairing mechanism.

\section{ACKNOWLEDGMENTS}

We are grateful to Jörg Schmalian for discussions. We acknowledge the Diamond Light Source for time on beamline I05 under Proposal No. SI16104 and thank all the beamline staff for technical support. We acknowledge DESY (Hamburg, Germany), a member of the Helmholtz Association HGF, for the provision of experimental facilities. Parts of this research were carried out at the P07 beamline at the PETRA III Synchrothron. C.E.M. acknowledges support from the Swiss National Science Foundation under Grants No. 200021-137783, No. P400P2_183890, and No. P2EZP2_175155. O.I., M.H., J.C., D.S., and J.C. acknowledge support from the Swiss National Science Foundation under Grants No. BSSGIO_155873 and No. PP00P2_150573, and through the Sinergia Network Mott Physics Beyond the Heisenberg Model. This work was supported by the Swiss National Science Foundation (Grant No. 200021_188413) and the Sino-Swiss Science and Technology Cooperation (Project No. IZLCZ2_170075).
[1] R. M. Fernandes, A. V. Chubukov, and J. Schmalian, What drives nematic order in iron-based superconductors? Nat. Phys. 10, 97 (2014).

[2] G. R. Stewart, Superconductivity in iron compounds, Rev. Mod. Phys. 83, 1589 (2011).

[3] H.-H. Kuo, J.-H. Chu, J. C. Palmstrom, S. A. Kivelson, and I. R. Fisher, Ubiquitous signatures of nematic quantum criticality in optimally doped Fe-based superconductors, Science 352, 958 (2016).

[4] S. Lederer, Y. Schattner, E. Berg, and S. A. Kivelson, Enhancement of Superconductivity near a Nematic Quantum Critical Point, Phys. Rev. Lett. 114, 097001 (2015).

[5] E. Fradkin, S. A. Kivelson, M. J. Lawler, J. P. Eisenstein, and A. P. Mackenzie, Nematic Fermi fluids in condensed matter physics, Annu. Rev. Condens. Matter Phys. 1, 153 (2010).
[6] J.-H. Chu, J. G. Analytis, K. D. Greve, P. L. McMahon, Z. Islam, Y. Yamamoto, and I. R. Fisher, In-plane resistivity anisotropy in an underdoped iron arsenide superconductor, Science 329, 824 (2010).

[7] J.-H. Chu, H.-H. Kuo, J. G. Analytis, and I. R. Fisher, Divergent nematic susceptibility in an iron arsenide superconductor, Science 337, 710 (2012).

[8] M. A. Tanatar, E. C. Blomberg, A. Kreyssig, M. G. Kim, N. Ni, A. Thaler, S. L. Bud'ko, P. C. Canfield, A. I. Goldman, I. I. Mazin, and R. Prozorov, Uniaxial-strain mechanical detwinning of $\mathrm{CaFe}_{2} \mathrm{As}_{2}$ and $\mathrm{BaFe}_{2} \mathrm{As}_{2}$ crystals: Optical and transport study, Phys. Rev. B 81, 184508 (2010).

[9] M. Yi, D. Lu, J.-H. Chu, J. G. Analytis, A. P. Sorini, A. F. Kemper, B. Moritz, S.-K. Mo, R. G. Moore, M. Hashimoto, W.-S. Lee, Z. Hussain, T. P. Devereaux, I. R. Fisher, and 
Z.-X. Shen, Symmetry-breaking orbital anisotropy observed for detwinned $\mathrm{Ba}\left(\mathrm{Fe}_{1-x} \mathrm{Co}_{x}\right)_{2} \mathrm{As}_{2}$ above the spin density wave transition, Proc. Natl. Acad. Sci. USA 108, 6878 (2011).

[10] Y. Kim, H. Oh, C. Kim, D. Song, W. Jung, B. Kim, H. J. Choi, C. Kim, B. Lee, S. Khim, H. Kim, K. Kim, J. Hong, and $\mathrm{Y}$. Kwon, Electronic structure of detwinned $\mathrm{BaFe}_{2} \mathrm{As}_{2}$ from photoemission and first principles, Phys. Rev. B 83, 064509 (2011).

[11] M. Yi, H. Pfau, Y. Zhang, Y. He, H. Wu, T. Chen, Z. R. Ye, M. Hashimoto, R. Yu, Q. Si, D.-H. Lee, P. Dai, Z.-X. Shen, D. H. $\mathrm{Lu}$, and R. J. Birgeneau, Nematic Energy Scale and the Missing Electron Pocket in FeSe, Phys. Rev. X 9, 041049 (2019).

[12] M. D. Watson, T. K. Kim, A. A. Haghighirad, N. R. Davies, A. McCollam, A. Narayanan, S. F. Blake, Y. L. Chen, S. Ghannadzadeh, A. J. Schofield, M. Hoesch, C. Meingast, T. Wolf, and A. I. Coldea, Emergence of the nematic electronic state in FeSe, Phys. Rev. B 91, 155106 (2015).

[13] M. Yi, D. H. Lu, R. G. Moore, K. Kihou, C.-H. Lee, A. Iyo, H. Eisaki, T. Yoshida, A. Fujimori, and Z.-X. Shen, Electronic reconstruction through the structural and magnetic transitions in detwinned NaFeAs, New J. Phys. 14, 073019 (2012).

[14] Y. Zhang, C. He, Z. R. Ye, J. Jiang, F. Chen, M. Xu, Q. Q. Ge, B. P. Xie, J. Wei, M. Aeschlimann, X. Y. Cui, M. Shi, J. P. $\mathrm{Hu}$, and D. L. Feng, Symmetry breaking via orbital-dependent reconstruction of electronic structure in detwinned $\mathrm{NaFeAs}$, Phys. Rev. B 85, 085121 (2012).

[15] S. Nandi, M. G. Kim, A. Kreyssig, R. M. Fernandes, D. K. Pratt, A. Thaler, N. Ni, S. L. Bud'ko, P. C. Canfield, J. Schmalian, R. J. McQueeney, and A. I. Goldman, Anomalous Suppression of the Orthorhombic Lattice Distortion in Superconducting $\mathrm{Ba}\left(\mathrm{Fe}_{1-x} \mathrm{Co}_{x}\right)_{2} \mathrm{As}_{2}$ Single Crystals, Phys. Rev. Lett. 104, 057006 (2010).

[16] Q. Huang, Y. Qiu, W. Bao, M. A. Green, J. W. Lynn, Y. C. Gasparovic, T. Wu, G. Wu, and X. H. Chen, NeutronDiffraction Measurements of Magnetic Order and a Structural Transition in the Parent $\mathrm{BaFe}_{2} \mathrm{As}_{2}$ Compound of FeAs-Based High-Temperature Superconductors, Phys. Rev. Lett. 101, 257003 (2008).

[17] M. Yi, Y. Zhang, Z.-X. Shen, and D. Lu, Role of the orbital degree of freedom in iron-based superconductors, npj Quantum Mater. 2, 57 (2017).

[18] W. Wang, Y. Song, C. Cao, K.-F. Tseng, T. Keller, Y. Li, L. W. Harriger, W. Tian, S. Chi, R. Yu, A. H. Nevidomskyy, and P. Dai, Local Orthorhombic lattice distortions in the paramagnetic tetragonal phase of superconducting $\mathrm{NaFe}_{1-x} \mathrm{Ni}_{x} \mathrm{As}$, Nat. Commun. 9, 3128 (2018).

[19] S. Katrych, K. Rogacki, A. Pisoni, S. Bosma, S. Weyeneth, R. Gaal, N. D. Zhigadlo, J. Karpinski, and L. Forró, $\mathrm{Pr}_{4} \mathrm{Fe}_{2} \mathrm{As}_{2} \mathrm{Te}_{1-x} \mathrm{O}_{4}$ : A layered FeAs-based superconductor, Phys. Rev. B 87, 180508(R) (2013).

[20] S. Katrych, A. Pisoni, S. Bosma, S. Weyeneth, N. D. Zhigadlo, R. Gaal, J. Karpinski, and L. Forró, $\mathrm{L}_{4} \mathrm{Fe}_{2} \mathrm{As}_{2} \mathrm{Te}_{1-x} \mathrm{O}_{4-y} \mathrm{~F}_{y}(\mathrm{~L}=$ $\mathrm{Pr}, \mathrm{Sm}, \mathrm{Gd})$ : A layered oxypnictide superconductor with $T_{c}$ up to 45 K, Phys. Rev. B 89, 024518 (2014).
[21] A. Pisoni, P. Szirmai, S. Katrych, B. Náfrádi, R. Gaál, J. Karpinski, and L. Forró, Magnetotransport studies of superconducting $\operatorname{Pr}_{4} \mathrm{Fe}_{2} \mathrm{As}_{2} \mathrm{Te}_{1-x} \mathrm{O}_{4}$, Phys. Rev. B 93, 094519 (2016).

[22] K. Kothapalli, A. E. Böhmer, W. T. Jayasekara, B. G. Ueland, P. Das, A. Sapkota, V. Taufour, Y. Xiao, E. Alp, S. L. Bud'ko, P. C. Canfield, A. Kreyssig, and A. I. Goldman, Strong cooperative coupling of pressure-induced magnetic order and nematicity in FeSe, Nat. Commun. 7, 12728 (2016).

[23] See Supplemental Material at http://link.aps.org/supplemental/ 10.1103/PhysRevResearch.3.023220 for further analysis and more detailed description of methods.

[24] U. Flechsig, L. Patthey, and T. Schmidt, Performance measurements at the SLS spectroscopy Beamline, in Synchrotron Radiation Instrumentation: Eighth International Conference on Synchrotron Radiation Instrumentation, edited by T. Warwick, J. Stöhr, H. A. Padmore, and J. Arthur, AIP Conf. Proc. No. 705 (AIP, New York, 2004). p. 316.

[25] M. Hoesch, T. K. Kim, P. Dudin, H. Wang, S. Scott, P. Harris, S. Patel, M. Matthews, D. Hawkins, S. G. Alcock, T. Richter, J. J. Mudd, M. Basham, L. Pratt, and P. Leicester et al., A facility for the analysis of the electronic structures of solids and their surfaces by synchrotron radiation photoelectron spectroscopy, Rev. Sci. Instrum. 88, 013106 (2017).

[26] P. Blaha, K. Schwarz, G. Madsen, D. Kvasnicka, J. Luitz, R. Laskowski, F. Tran, and L. D. Marks, Wien $2 k$ : An Augmented Plane Wave + Local Orbitals Program for Calculating Crystal Properties (Karlheinz Schwarz, Techn. Universität Wien, Austria, 2018).

[27] P. Blaha, K. Schwarz, F. Tran, R. Laskowski, G. K. H. Madsen, and L. D. Marks, WIEN2k: An APW+lo program for calculating the properties of solids, J. Chem. Phys. 152, 074101 (2020).

[28] G. J. McIntyre, A. Renault, and G. Collin, Domain and crystal structure of superconducting $\mathrm{Ba}_{2} \mathrm{YCu}_{3} \mathrm{O}_{8-\delta}$ at 40 and $100 \mathrm{~K}$ by single-crystal neutron diffraction, Phys. Rev. B 37, 5148 (1988).

[29] B. A. Frandsen, K. M. Taddei, M. Yi, A. Frano, Z. Guguchia, R. Yu, Q. Si, D. E. Bugaris, R. Stadel, R. Osborn, S. Rosenkranz, O. Chmaissem, and R. J. Birgeneau, Local Orthorhombicity in the Magnetic $C_{4}$ Phase of the Hole-Doped Iron-Arsenide Superconductor $\mathrm{Sr}_{1-x} \mathrm{Na}_{x} \mathrm{Fe}_{2} \mathrm{As}_{2}$, Phys. Rev. Lett. 119, 187001 (2017).

[30] P. Zhang, P. Richard, T. Qian, Y.-M. Xu, X. Dai, and H. Ding, A precise method for visualizing dispersive features in image plots, Rev. Sci. Instrum. 82, 043712 (2011).

[31] M. H. Christensen, R. M. Fernandes, and A. V. Chubukov, Orbital transmutation and the electronic spectrum of FeSe in the nematic phase, Phys. Rev. Research 2, 013015 (2020).

[32] M. D. Watson, T. K. Kim, L. C. Rhodes, M. Eschrig, M. Hoesch, A. A. Haghighirad, and A. I. Coldea, Evidence for unidirectional nematic bond ordering in FeSe, Phys. Rev. B 94, 201107(R) (2016).

[33] M. D. Watson, A. A. Haghighirad, L. C. Rhodes, M. Hoesch, and T. K. Kim, Electronic anisotropies revealed by detwinned angle-resolved photo-emission spectroscopy measurements of FeSe, New J. Phys. 19, 103021 (2017). 